\title{
Noninvasive photoacoustic microscopy of methemoglobin in vivo
}

\section{Min Tang, Yong Zhou, Ruiying Zhang, Lihong V. Wang}

Min Tang, Yong Zhou, Ruiying Zhang, Lihong V. Wang, "Noninvasive photoacoustic microscopy of methemoglobin in vivo," Proc. SPIE 9323, Photons Plus Ultrasound: Imaging and Sensing 2015, 93233K (11 March 2015); doi: $10.1117 / 12.2076692$

SPIE. Event: SPIE BiOS, 2015, San Francisco, California, United States 


\title{
Noninvasive photoacoustic microscopy of methemoglobin in vivo
}

\author{
Min Tang†, Yong Zhou†, Ruiying Zhang, and Lihong V. Wang* \\ Optical Imaging Laboratory, Department of Biomedical Engineering, Washington University in \\ St. Louis, One Brookings Drive, St. Louis, MO 63130, USA \\ *Address all correspondence to: lhwang@wustl.edu
}

\begin{abstract}
Various causes can lead to methemoglobinemia, and it has the potential to be confused with other diseases. In vivo measurements of methemoglobin have significant applications in the clinics. We quantified the average and the distributed percentage of methemoglobin both in vitro and in vivo using photoacoustic microscopy (PAM). Based on the absorption spectra of methemoglobin, oxyhemoglobin, and deoxyhemoglobin, three wavelengths were chosen to differentiate methemoglobin from the others. We imaged the methemoglobin percentage in microtubes that mimicked blood vessels as a phantom experiment. The methemoglobin concentrations calculated from the photoacoustic signals were in accordance with the preset concentrations. We also demonstrated the ability of PAM to quantitatively image methemoglobin distribution in vivo in a mouse ear.
\end{abstract}

Keywords: photoacoustic microscopy, methemoglobin, distributed percentage in vivo. $\dagger$ The authors contributed equally to this work.

\section{Introduction}

Methemoglobin is a type of hemoglobin that contains oxidized iron atoms in the ferric state and is incapable of transporting oxygen to body tissues $[1,2]$. Elevated methemoglobin levels in the blood stream cause tissue hypoxia and can lead to tachycardia, stupor, and impaired respiration. Even death may occur in humans when the methemoglobin level is over $70 \%$ [3]. Measuring the methemoglobin level, both the average concentration and spatial distribution, has extensive clinical applications.

The motivation of this work is to quantitatively image methemoglobin distribution in vivo with PAM. 3D photoacoustic microscopy (PAM) has demonstrated its potential in structural and functional imaging [4-9]. We first showed that PAM was capable of detecting methemoglobin distributions in vessel-mimicking phantoms. Then, we imaged the methemoglobin distribution in a mouse ear in vivo during a period of induction and recovery of methemoglobin. We demonstrated for the first time that PAM can quantitatively image the spatial distribution of methemoglobin in vivo.

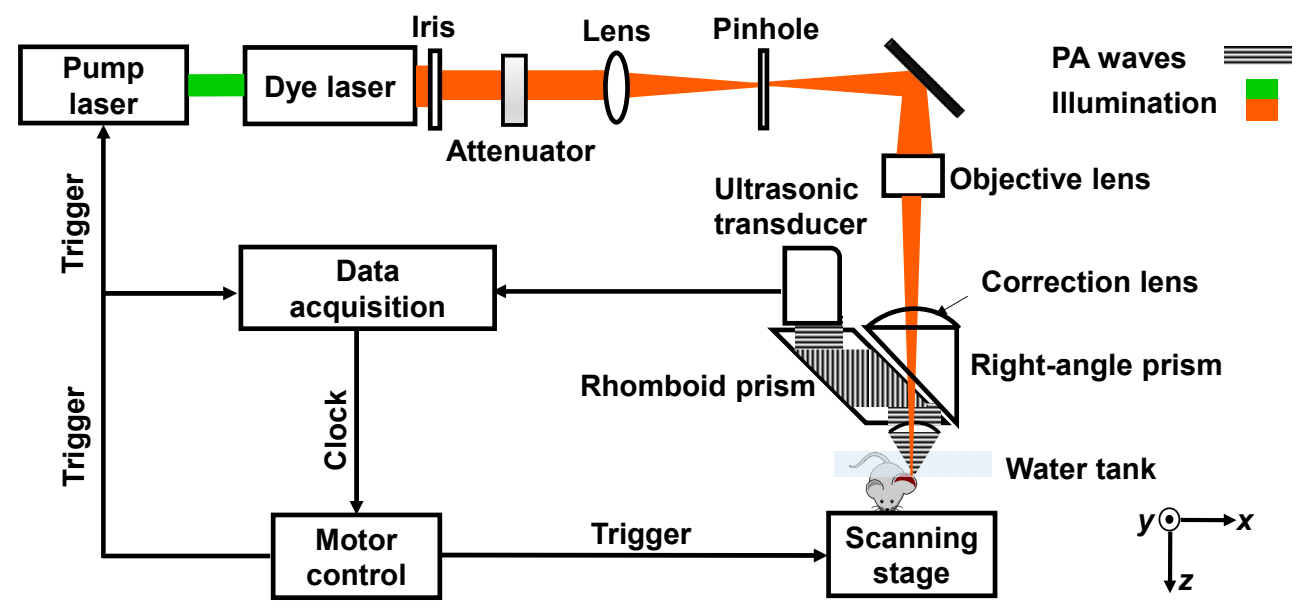

Fig. 1 Schematic of PAM system. 


\section{Experimental system}

An optical-resolution PAM (OR-PAM) system [10-12] was used to measure methemoglobin distribution, as shown in Fig. 1. The light was produced by a tunable dye laser (CBR-D, Sirah GmbH) pumped by an Nd:YLF laser (INNOSAB, Edgewave $\mathrm{GmbH}$ ) at a $1 \mathrm{kHz}$ pulse rate. The laser beam was focused by a lens, filtered by a pinhole, reflected by a mirror, and then focused into the object to be imaged by an objective lens. An acoustic-optical beam combiner containing two prisms with a layer of silicone oil in between provided the acoustic optical coaxial alignment. Then a wideband ultrasonic transducer (V214-BC, Panametrics-NDT Inc.) placed confocally with the optical objective lens detected the acoustic signals. A depth-resolved one-dimensional (1D) photoacoustic A-line signal was obtained from each laser pulse. By raster scanning the object in two dimensions and performing maximum amplitude projections (MAP) of the A-line signals, a two-dimensional MAP PAM image of the sample was obtained.

\section{Phantom experiment}

Induction of methemoglobin formation was achieved by adding Sodium nitrite (237213-500G, Sigma-Aldrich) powder into the lysed bovine blood (905, Quad-Five) samples. To induce a level of $100 \%$ methemoglobin, $24.3 \mathrm{~g}$ sodium nitrite powder was added to $50 \mathrm{ml}$ of bovine blood. Then blood samples with methemoglobin concentrations of $20 \%, 40 \%, 60 \%$, and $80 \%$ were obtained by mixing $2 \mathrm{ml}, 4 \mathrm{ml}, 6 \mathrm{ml}$, and $8 \mathrm{ml}$ of the $100 \%$ methemoglobin blood with $8 \mathrm{ml}, 6 \mathrm{ml}, 4 \mathrm{ml}$, and $2 \mathrm{ml}$ of pure bovine blood, respectively. The mixtures were then separately injected into microtubes (60985-700, VWR) made of platinum-cured silicone, with $300-\mu \mathrm{m}$ inner diameters and $600-\mu \mathrm{m}$ outer diameters, to mimic blood with different methemoglobin concentrations in blood vessels.

Methemoglobin concentrations were measured by PAM in tissue-mimicking phantoms. Samples with different induced methemoglobin levels were prepared with the method described above. Five microtubes filled with bovine blood of average methemoglobin concentrations of $23 \%, 41 \%, 58 \%, 76 \%$, and $89 \%$ were used. Wavelengths 610 $\mathrm{nm}, 620 \mathrm{~nm}$, and $630 \mathrm{~nm}$ were selected for PAM imaging, with a scanning area of $4 \mathrm{~mm}$ by $2 \mathrm{~mm}$. The normalized PAM images obtained at each wavelength are shown in Fig. 2(a). We showed that the PAM results averaged within each tube agreed well with the preset concentrations, as shown in Fig. 2(b).
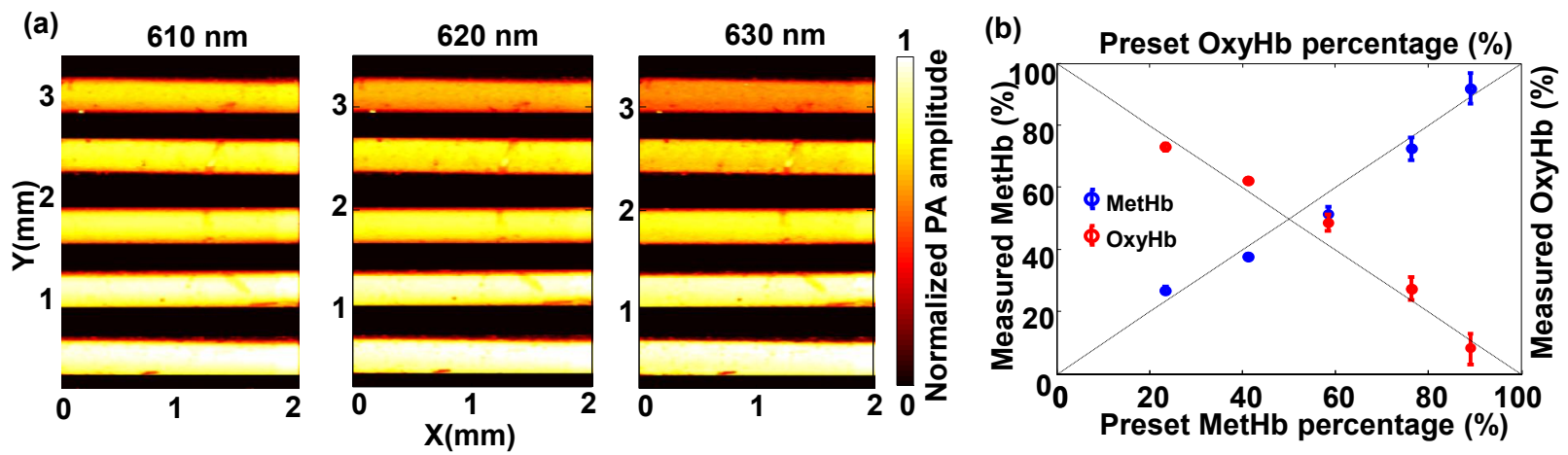

Fig. 2 (a) Maximum-amplitude projection (MAP) PAM images of tubes filled with different concentrations of methemoglobin in lysed bovine blood at $610 \mathrm{~nm}, 620 \mathrm{~nm}$, and $630 \mathrm{~nm}$. (b) The photoacoustically measured methemoglobin and oxyhemoglobin percentage averaged within each tube versus the preset value. The preset percentages were measured with a spectrophotometer. Lines: ideal fit if the photoacoustically measured and preset methemoglobin percentages are identical.

\section{In vivo experiment}

Injection of $60 \mu \mathrm{l}$ well-mixed sodium nitrite solution (10 $\mathrm{mg}$ sodium nitrite/ $1 \mathrm{ml}$ de-ionized water) through the jugular vein of a mouse induced methemoglobin formation. The volume and concentration were calculated based on the Kohn's Model [13]. The blood vessels of the mouse ear were then imaged by the PAM system at multiple selected optical wavelengths. 
The mouse ear was imaged by PAM consecutively for 90 minutes to include both the methemoglobin-induction activity and the recovery activity of the mouse's self-reductive systems. An area of $4 \mathrm{~mm}$ by $4 \mathrm{~mm}$ was first scanned with wavelength of $610 \mathrm{~nm}$ to show the blood vessels on the mouse's ear (Fig. 3(a)), then a $1 \mathrm{~mm}$ by $0.5 \mathrm{~mm}$ area (the rectangular area in Fig. 3(a)) was imaged at the three wavelengths once every 6 minutes to monitor the change in methemoglobin level during the 90 minutes. The change in methemoglobin concentration measured by PAM was in accordance with the prediction of the Kohn model (Fig. 3(b)) [13].

(a)

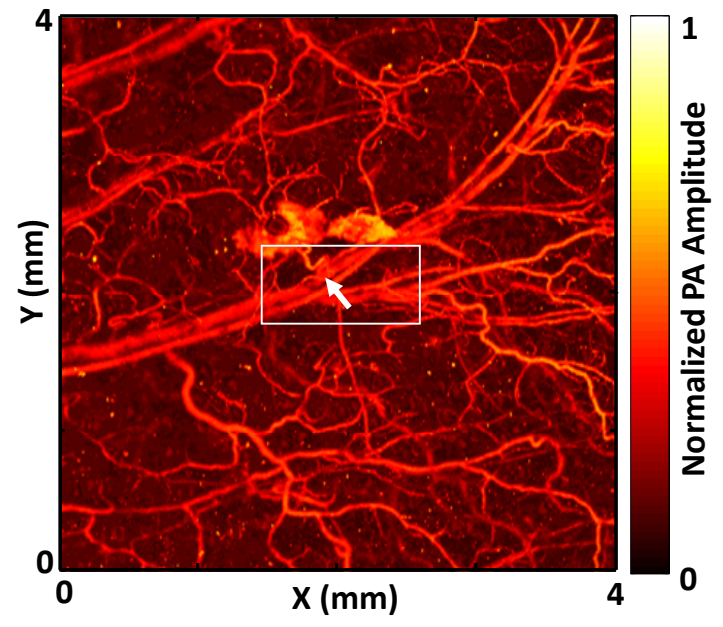

(b)

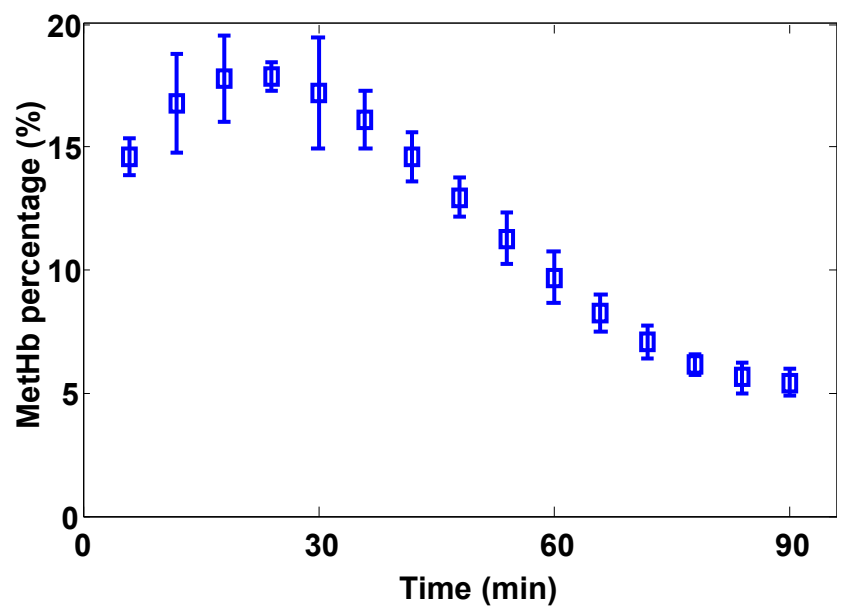

Fig. 3 (a) MAP PAM image of blood vessels of a mouse ear. The vessel indicated by the arrow in the white rectangle was monitored to calculate the methemoglobin level during the induction and self-recovery periods. (b) Time course of methemoglobin percentage after intravenous injection of sodium nitrite. Data are presented as mean \pm standard error of mean.

\section{Conclusions}

To the best of our knowledge, this is the first study to use PAM to quantitatively image the methemoglobin in vivo. The methemoglobin concentration and distribution were imaged by PAM in tissue-mimicking phantoms at multiple wavelengths. We also measured the methemoglobin concentration change in vivo after induction of methemoglobinemia in a mouse. After intravenous injection of sodium nitrite solution, the methemoglobin level measured in the blood vessels of the mouse ear increased to $\sim 18 \%$ and peaked around 24 minutes after injection. The methemoglobin concentration gradually decreased to the normal level by the end of the measurement. This trend of change accords with the prediction of the Kohn Model. Our study demonstrated PAM's ability to quantify methemoglobin levels in vivo.

\section{Acknowledgments}

The authors gratefully acknowledge the suggestions made by the reviewers of this manuscript, and by Prof. James Ballard at Washington University in St. Louis. This research was supported in part by National Institutes of Health grants DP1 EB016986, R01 CA186567, U01 NS090579, R01 EB016963, R01 EB010049, R01 CA157277, S10 RR028864, S10 RR026922, and R01 CA159959 as well as National Science Foundation grant 1255930. L.W. has a financial interest in Microphotoacoustics, Inc. and Endra, Inc., which, however, did not support this work.

\section{References}

1. N. E. Camp, "Methemoglobinemia," Journal of emergency nursing: JEN : official publication of the Emergency Department Nurses Association 33(2), 172-174 (2007).

2. $\quad$ S. Bradberry, "Methaemoglobinaemia," Medicine 40(2), 59-60 (2012).

3. H. U. Rehman, "Methemoglobinemia," The Western journal of medicine 175(3), 193-196 (2001). 
4. Y. Zhou, X. Yi, W. Xing, S. Hu, K. Maslov, and L. V. Wang, "Microcirculatory changes identified by photoacoustic microscopy in patients with complex regional pain syndrome type I after stellate ganglion blocks," J. Biomed. Opt. 19(8), 086017 (2014).

5. Y. Zhou, J. Liang, K. I. Maslov, and L. V. Wang, "Calibration-free in vivo transverse blood flowmetry based on cross correlation of slow time profiles from photoacoustic microscopy," Opt. Lett. 38(19), 38823885 (2013).

6. L. V. Wang, and S. Hu, "Photoacoustic tomography: in vivo imaging from organelles to organs," Science 335(6075), 1458-1462 (2012).

7. Y. Zhou, W. Xing, K. I. Maslov, Lynn A. Cornelius, and L. V. Wang, "Handheld photoacoustic microscopy to detect melanoma depth in vivo," Opt. Lett. 39(16), 4731-4734 (2014).

8. Y. Zhou, G. Li, L. Zhu, C. Li, L. A. Cornelius, and L. V. Wang, "Handheld photoacoustic probe to detect both melanoma depth and volume at high speed in vivo," J. Biophotonics, 1-7 (2015).

9. Y. Zhou, C. Zhang, D. Yao and L. V. Wang, "Photoacoustic microscopy of bilirubin in tissue phantoms," J. Biomed. Opt. 17(12), 126019 (2012).

10. P. Hai, J. Yao, K. I. Maslov, Y. Zhou, and L. V. Wang, "Near-infrared optical-resolution photoacoustic microscopy," Optics letters 39(17), 5192-5195 (2014).

11. Y. Zhou, J. Yao, and L. V. Wang, "Optical clearing-aided photoacoustic microscopy with enhanced resolution and imaging depth," Optics letters 38(14), 2592-2595 (2013).

12. Y. Zhou, J. Yao, K. I. Maslov, and L. V. Wang, "Calibration-free absolute quantification of particle concentration by statistical analyses of photoacoustic signals in vivo," J. Biomed. Opt. 19(3), 037001 (2014).

13. M. C. Kohn, R. L. Melnick, F. Ye, and C. J. Portier, "Pharmacokinetics of sodium nitrite-induced methemoglobinemia in the rat," Drug metabolism and disposition: the biological fate of chemicals 30(6), 676-683 (2002). 